\title{
Optimal Bus-Bridging Service under a Metro Station Disruption
}

\author{
Haodong Yin, ${ }^{1}$ Jianjun Wu $\left(\mathbb{D},{ }^{1,2}\right.$ Huijun Sun $\left(\mathbb{D},{ }^{3}\right.$ Yunchao Qu, ${ }^{1}$ Xin Yang, ${ }^{1}$ and Bo Wang ${ }^{4}$ \\ ${ }^{1}$ State Key Laboratory of Rail Traffic Control and Safety, Beijing Jiaotong University, Beijing 100044, China \\ ${ }^{2}$ Key Laboratory of Transport Industry of Big Data Application Technologies for Comprehensive Transport, Ministry of Transport, \\ Beijing Jiaotong University, Beijing 100044, China \\ ${ }^{3}$ MOE Key Laboratory for Urban Transportation Complex Systems Theory and Technology, Beijing Jiaotong University, \\ Beijing 100044, China \\ ${ }^{4}$ Beijing Municipal Commission of Transport, Beijing 100073, China
}

Correspondence should be addressed to Jianjun Wu; jjwul@bjtu.edu.cn

Received 18 March 2018; Revised 10 May 2018; Accepted 22 May 2018; Published 5 July 2018

Academic Editor: Zhiyuan Liu

Copyright (c) 2018 Haodong Yin et al. This is an open access article distributed under the Creative Commons Attribution License, which permits unrestricted use, distribution, and reproduction in any medium, provided the original work is properly cited.

\begin{abstract}
A station disruption is an abnormal operational situation that the entrance or exit gates of a metro station have to be closed for a certain of time due to an unexpected incident. The passengers' travel behavioral responses to the alternative station disruption scenarios and the corresponding controlling strategies are complex and hard to capture. This can lead to the hardness of estimating the changes of the network-wide passenger demand, which is the basis of carrying out a response plan. This paper will establish a model to solve the metro station disruption problem by providing optimal additional bus-bridging services. Two main contributions are made: (1) a three-layer discrete choice behavior model is developed to analyze the dynamic passenger flow demand under station disruption; and (2) an integrated algorithm is designed to manage and control the station disruption crisis by providing additional bus-bridging services with the objective of minimizing the total travel time of affected passengers and the operating cost of bridging-buses. Besides, the multimodal transport modes, including metro, bridging-bus, shared-bike, and taxi, are considered as passengers' alternative choices in face of the station disruption. A numerical study based on the Beijing metro network shows that additional bus-bridging services can significantly eliminate the negative impact of the station disruption.
\end{abstract}

\section{Introduction}

Station disruption, also called station closure, is an abnormal and usually unplanned operational situation in which operators must close the entrances or exits of a metro station for various reasons, such as unexpected incidents or taking steps to avoid overcrowding [1]. In these situations, passengers cannot use the closed station as their departure station or destination stations during the closure time. Generally, disruption means a serious deviation from the planned operations in the rail transit context [2-6]. Station disruption is a typical case of metro disruption, and it can strongly affect both the service and demand of the metro system. At the service level, a station disruption means that the trains, which are planning to stop at the closed station, have to adopt other alternative routes to detour the closed station, e.g., passing the closed station without stopping. At the demand level, the station disruption may cause significant changes in passenger flow demand at the closed stations and nearby stations.

To our knowledge, limited existing studies are available related to the topic of station disruption. Silva et al. [7] proposed a data-driven statistical method to determine the effect of station disruptions on the macroscopic passenger flow demand based on the smart card data from the day of the station disruption and the historical data. This approach was proved to be effective for identifying the impact of a station disruption, but it is not applicable for predicting the impact of a station disruption that does not actually occur or is about to occur. Another effective mathematical model that could capture the behavior of passengers affected by a station disruption was proposed for analyzing alternative disruption scenarios and their likely outcomes in our previous study [1]. But this model ignored the randomness of the passengers' choice behaviors and the influence of the controlling strategies 
on passenger behaviors. Some other researchers studied the impacts of traffic incidents on road traffic, which could provide references for this study in metro system $[8,9]$.

Although we can capture the dynamic influence of the station disruption on passenger flow demand to some extent, how to minimize the severity of potential negative impacts of the station disruption by carrying the affected passengers to the destination with high service quality and low operation cost is still unsolved. Rescheduling timetables plays a limited role in solving the station disruption problem [10-18], because the station disruption defined in this paper does not interrupt the trains running process on the metro lines. But the bus-bridging service has been recognized as one of the most critical components of effective disruption responses in metro networks. Kepaptsoglou and Karlaftis [19] firstly proposed a methodological framework for planning and designing an efficient bus-bridging network. Wang et al. [20] explored the demand modeling problem for busbridging disruption in metro services based on the theory of compound Poisson processes and formulated it as a bulk queuing problem involving balking and reneging. Jin et al. [21] introduced a localized metro-bus integration approach that aimed at enhancing the urban transit networks resilience to disruption. Based on the previous researches, Jin et al. [22] presented an optimization-based approach of designing an optimal bus-bridging service for network disruptions by considering the commuter travel demand at the time of the disruption. But the travel demand was estimated from the historical data. In other words, the dynamic responses of passengers to the disruption were not considered.

In this paper, we attempt to manage and control the station disruption by providing additional bus-bridging services for passengers. Our contributions mainly consist of the following two parts: (1) a three-layer discrete choice behavior model is developed to analyze the dynamic passenger flow demand under station disruption; and (2) an integrated algorithm is designed to manage and control the station disruption crisis by providing additional busbridging services with the objective of minimizing the total travel time of affected passengers and the operating cost of bridging-buses. Besides, the multimodal transport modes, including metro, bridging-bus, shared-bike, and taxi, are considered as passengers' alternative choices in face of the station disruption.

The paper is organized as follows: Section 2 presents the detailed description of station disruption problem and the integrated optimization approach based on agent-based simulation. The case study results are reported in Section 3. Finally, conclusions are given in Section 4.

\section{Bus-Bridging Service for the Station Disruption Problem}

In this section, we firstly introduce the metro station disruption problem in the metro network. Then, we describe this problem with an optimization approach integrated with a three discrete choice behavior model. In this approach, an optimal bus-bridging service plan is generated by a binary integer programming model. Besides, the passengers' response to the bus-bridging information and its randomness are also formulated in the behavior model. Finally, a solution algorithm is developed to solve the problem.

2.1. Problem Description. Consider a scenario that an emergent event causes a temporary closure of a station in the metro network. We also assume that the metro operator can accurately estimate the closure duration time and then inform the network-wide passengers immediately for simplifying the problem. Not only passengers whose departure station or destination station is the closed station are affected, but also the passengers around the closed station are potentially affected. As illustrated in Figure 1, station $B$ is temporarily closed. The affected passengers planning to travel by metro have to alter to other alternative transport modes, such as taxi, shared-bike, bridging-bus, and walking.

Consequently, for passengers, they cannot board or alight the trains passing through without stopping at the closed station $B$. For the passengers with their departure stations as station $B$, they may choose other alternative stations near the closed station as their new departure stations, wait at the closed station for recovery, or take another transit mode to finish their travels. For the passengers that station $B$ is their planned destinations, they have to decide whether to choose an alternative destination station or take another transit mode. The passengers make travel decisions according to their travel experiences and the released guidance information from public transit operators.

For metro operators, they must quickly carry out a responsive plan to replenish the services for the affected passengers. A common strategy is to restore the connectivity between the closed station and the rest of the metro network by running bus-bridging services. And, in station disruption problem, we consider designing the optimal direct busbridging routes between the closed station and the rest of the rail stations with an objective of minimizing total travel time of affected passengers and the operation cost.

In planning for responses to station disruption, challenges are to estimate the dynamic passenger demand interacting with the operational information and then to decide which stations should be connected with the closed station by bus-bridging services. Notice that when bridging-buses are operated between the closed station and nearby stations, the passengers will also decide whether to choose it to finish their travels or not. Therefore, it is a complicated decision problem: which stations among the network-wide stations should be selected to connect with the closed station with the objective of minimizing total travel time of affected passengers and the operating cost of bridging-buses, considering the dynamics of passenger behavior and demand. To describe and solve the station disruption problem, a hybrid optimization approach based on binary integer programming and passenger behavior simulation is constructed. And, in order to simplify the problem, we constrain our research to the scenario where only a single station is closed.

The basic framework of the model is shown in Figure 2. The objective function of the optimization model is minimizing the total travel time of affected passengers and the 


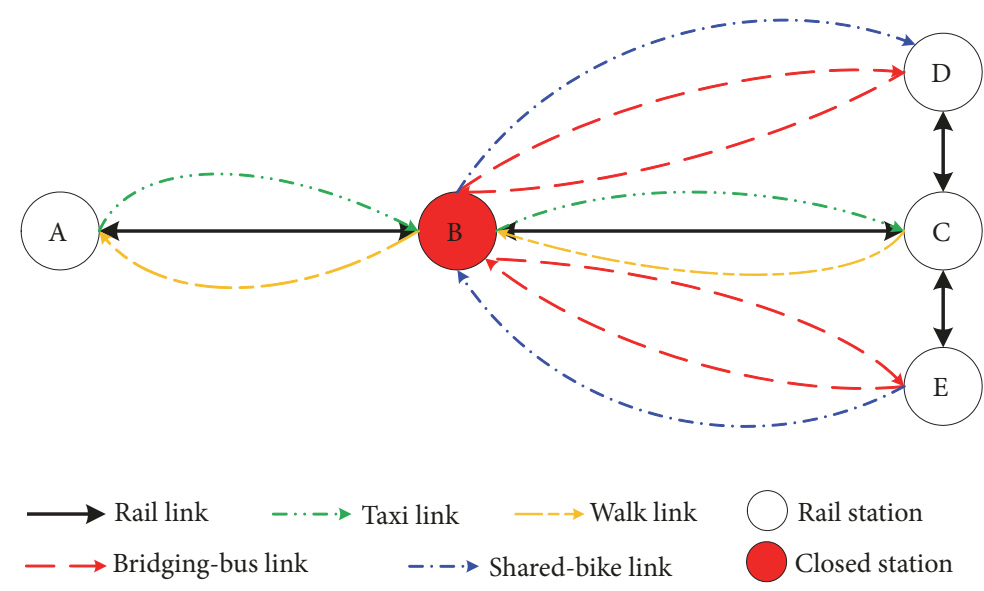

FIgURE 1: Illustrative example of station disruption problem in metro networks.

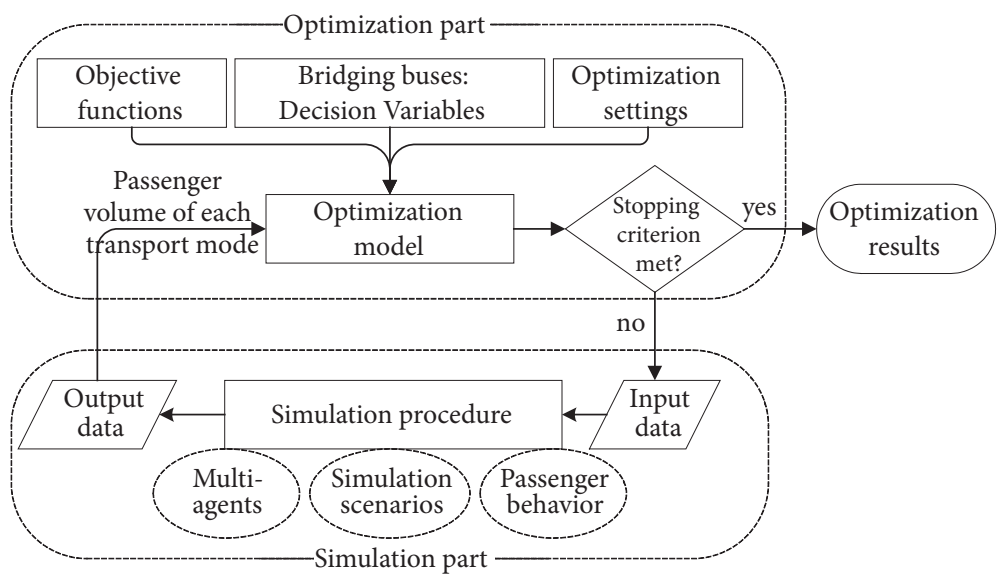

FIGURE 2: The basic framework of station disruption controlling model.

operating cost of bridging-buses. The simulation procedure is used to describe the feedback of the passenger to the bridging-buses and then to output the dynamic traffic flow for the optimization part. While the urban rail transit system involves large-scale passengers and their dynamic travel decisions under station disruption, the passenger behavior cannot be described by the precise mathematical method. Therefore, an agent-based passenger behavior simulation model is considered to describe the behavioral response of the passengers to the station disruption scenario and its corresponding bus-bridging schemes.

2.2. Model Notations. Main notations used in the paper are listed as follows. All variables are assumed to be integer numbers to satisfy the engineering requirements.

\section{Parameters and Sets}

$G(\boldsymbol{S}, \boldsymbol{A})$ : directed graph to denote the metro network. $S$ indicates the station set, and $A$ indicates the set of directed arcs.

$i, j, s$ : index of a station, $i, j, s \in S$.

$a$ : index of an directed arc, $a \in A$.
$M$ : the vector of transport modes, $M=$ \{bus, metro, taxi, bike, walk\}, and the bus stands for the bridging-bus.

$M^{\prime}$ : the vector of transport modes except for metro, $M^{\prime}=$ bus, taxi, bike, walk $\}$.

$m$ : index of transport modes, $m \in M$.

$C_{i}^{m}$ : the carrying capacity of the transport mode $m$ departing from the station $i$.

$d_{i j}^{m}$ : distance of the shortest path from station $i$ to station $j$ by the transport mode $m$.

$t_{i j}^{m}$ : travel time of the shortest path from station $i$ to station $j$ by the transport mode $m$.

$b$ : index of the closed station, $b \in S$.

$N$ : the total number of the available bridging-buses.

\section{Decision Variables}

$P$ : set of passengers in the current metro system.

$P_{i j}^{t}$ : set of passengers from station $i$ to station $j$ at time $t, P_{i j}^{t} \subset P$. 
$p$ : index of the passenger with the origin station $i$, destination station $j$, and departure time of time $t$, $p \in P_{i j}^{t}$.

$\alpha_{m}$ : the value of the travel time of urban transport mode $m$, which can be found in local studies in China.

$\beta_{m}$ : the comfort penalty parameter of urban transport mode $m$, and there exits $\beta_{\text {walk }}>\beta_{\text {bike }}>\beta_{\text {bus }}>\beta_{\text {taxi }}$ according to our common sense.

$f_{i j}^{m}$ : the fee for taking transport mode $m$ with the shortest path from station $i$ to station $j$.

$c_{i j}^{m, t}$ : the generalized cost of taking transport mode $m$ from station $i$ to station $j$ at time $t$ considering the travel time and price. $v_{i}^{m, t}:$ the total number of passengers taking transport mode $m$ at station $i$ at time $t$.

\section{Intermediate Variables}

$x_{i}(t)$ : indicates that whether operating bridging-buses from the closed station $b$ to station $i$ at time $t$ and then back to closed station at time $t+t_{b, i}^{\text {bus }} 1$ denotes "YES"; 0 stands for "NO."

\section{Station Disruption Parameters}

$\psi_{s}^{p}$ : the main decision variable denotes whether station $s$ should be chosen as the alternative origin or destination station for the passenger $p$, where

$$
\psi_{s}^{p}= \begin{cases}1, & \text { if station } s \text { is selected by the passenger } p \text { as the replaceable origin/destination station; } \\ 0, & \text { otherwise. }\end{cases}
$$

$z_{i j}^{m, p}:$ the decision variable denotes whether the transport mode $m$ should be the alternative transport mode from station $i$ to station $j$ at time $t$ for the passenger $p$, where

$$
z_{i j}^{m, p}= \begin{cases}1, & \text { if the tranport mode } m \text { is selected as the replaceble transport mode and } i \neq j ; \\ 0, & \text { otherwise. }\end{cases}
$$

$\gamma_{i}$ : the constant parameter denoting whether station $i$ is closed for the passenger $p . \gamma_{i}$ equals 1 when station $i$ is out of service and is 0 otherwise.

$t_{i}^{\text {start }}$ : the beginning time of the disruption of station $i$.

$$
\begin{aligned}
& t_{i}^{e n d} \text { : the actual ending time of the disruption of station } \\
& i \text {. } \\
& e_{i j}^{m, t} \text { : the extra journey time cost of metro transit from } \\
& \text { station } i \text { to station } j \text { at time } t \text { due to the station } \\
& \text { disruption: }
\end{aligned}
$$

$$
e_{i j}^{m, t}= \begin{cases}t_{i}^{e n d}-t, & \text { if station } i \text { is closed and } m=\text { metro, } t_{j}^{\text {end }}>t \\ t_{j}^{e n d}-t-t_{i j}^{m, t}, & \text { if station } j \text { is closed and } m=\text { metro, } t_{j}^{\text {end }}>t \\ 0, & \text { otherwise }\end{cases}
$$

2.3. Model Formulations. The proposed integrated optimization model includes two parts. The optimization part determines which stations should be set as a bridge with the closed station while minimizing the operating cost of bridging-buses and the total travel time of affected passengers. However, the behavior model part is used to capture the passenger choice behavior response to station disruption with a three-layer discrete choice model, determining which alternative travel strategy, which alternative origin or destination, and which replaceable transport mode will be chosen by the affected passengers.
2.3.1. Optimization Model. The optimization model is used to decide which stations should be connected to the closed station around the network with a goal of minimizing the operating cost of bridging-buses and the total travel time of affected passengers. The model can be written as follows:

$\min \quad \mathrm{Z}=2 \mu \sum_{i \in S, i \neq b}\left(x_{i}(t) * d_{b, i}^{b u s}\right)+\gamma \sum_{i \in S} g\left(x_{i}\right)$

s.t. $g\left(x_{i}\right)$ 


$$
\begin{aligned}
& =\sum_{p \in P_{b, i}^{t}} \sum_{s \in S} \psi_{s}^{p}\left(\sum_{m \in M^{\prime}} z_{b, s}^{m, p} t_{b, s}^{m}+t_{s, i}^{m e t r o}+e_{s, i}^{m e t r o, t}\right) \\
& \quad+\sum_{p \in P_{i, b}^{t}} \sum_{s \in S} \psi_{s}^{p}\left(t_{i, s}^{m e t r o}+e_{i, s}^{m e t r o, t}+\sum_{m \in M^{\prime}} z_{s, b}^{m, p} t_{s, b}^{m}\right) \\
& \sum_{s \in S} \psi_{s}^{p}=1, \quad \forall p \\
& \sum_{m \in M^{\prime}} z_{i j}^{m, p} \leq 1, \quad \forall p, i \neq j \in S \\
& \sum_{i \in S} \sum_{p \in P_{b, i}^{t}} \sum_{s \in S} \psi_{s}^{p} z_{b, s}^{m, p} \leq C_{b}^{m}, \quad \forall m \in M^{\prime} \\
& \sum_{i \in S} \sum_{p \in P_{i, b}^{t}} \psi_{s}^{p} z_{s, b}^{m, p} \leq C_{s}^{m}, \quad \forall m \in M^{\prime} \\
& x_{i}(t)=0,1, \quad \forall i \neq b \in S
\end{aligned}
$$

As indicated in (4), the objective function contains two parts: The first part is the operating cost of bridging-buses, running between the disrupted station and other stations. The second part is the total travel time of all affected passengers travelling with various transport modes.

Equation (5) defines the function to calculate the total travel time of all affected passengers. In the function, the affected passengers are defined as two types: one is the passenger whose planned origin station is closed, and the other is the passenger whose planned destination is closed.
The travel time of two types of passengers is calculated differently.

Equations (6)-(7) indicate the constraints of the passenger behavioral choices. Equation (6) shows that an alternative origin or destination station will be chosen for a particular passenger $p$. Constraint (7) indicates that one new transport mode will bridge the invalid origin station and the new replaceable station, or the new destination station to the invalid destination station.

Equations (8)-(9) indicate the capacity constraints of multimodal transport resources. Constraint (8) denotes the capacity constraints of the transport mode $m$ at the closed station $b$. Constraint (9) denotes the capacity constraints of the transport mode $m$ at the closed station $s$.

Equation (10) shows the decision variables are binary.

2.3.2. Passenger Behavior Model. The lower model is to capture the behavioral responses to the station disruption and its corresponding bus-bridging strategies and considering the randomness of the choice behavior on alternative stations or transport modes, e.g., bridging-buses, taxis, metro, sharedbicycles, and walking.

Generate the Optimal Travel Decision. For a passenger $p \in$ $P_{O, D}^{t}$, the objective of passenger behavior model is to minimize the generalized travel cost of making a new travel decision for the station disruption. The decision variables are $\psi_{s}^{p}$ and $z_{i j}^{m, p}$.

$$
\text { Objective min } \sum_{i \in\{O, D\}} \gamma_{i}^{p} J_{i}^{p}
$$

Subject to

$$
\begin{aligned}
& J_{O}^{p}=\sum_{s \in S} \psi_{s}^{p}\left(\sum_{m \in M^{\prime}} z_{i j}^{m, p} c_{O, s}^{m, t}+c_{s, D}^{m e t r o, t}\right), \quad \forall t, p, \text { if } O \text { is out of service } \\
& J_{D}^{p}=\sum_{s \in S} \psi_{s}^{p}\left(c_{O, s}^{m e t r o, t}+\sum_{m \in M^{\prime}} z_{i j}^{m, p} c_{s, D}^{m, t}\right), \quad \forall t, p, \text { if } D \text { is out of service } \\
& \gamma_{O}^{p}+\gamma_{D}^{p}=1, \quad \forall p \\
& \sum_{m \in M^{\prime}} z_{i j}^{m, p}=1, \quad \forall p, i \neq j \\
& \sum_{s \in S} \psi_{s}^{p}=1, \quad \forall p
\end{aligned}
$$

$$
\begin{aligned}
& c_{i j}^{m, t}=\left\{\begin{array}{ll}
W, & \text { if } v_{i}^{m, t}>C_{i}^{m} ; \\
\beta_{m}\left(\alpha_{m}\left(t_{i j}^{m}+e_{i j}^{m, t}\right)+f_{i j}^{m}\right)+\left(1-x_{i}(\mathrm{t})\right) W, & \text { if } m={ }^{\prime} b u s^{\prime}, j=b ; \\
\beta_{m}\left(\alpha_{m}\left(t_{i j}^{m}+e_{i j}^{m, t}\right)+f_{i j}^{m}\right)+\left(1-x_{j}(\mathrm{t})\right) W, & \text { if } m={ }^{\prime} b u s^{\prime}, i=b ; \\
\beta_{m}\left(\alpha_{m}\left(t_{i j}^{m}+e_{i j}^{m, t}\right)+f_{i j}^{m}\right), & \text { elsewhere. }
\end{array} \quad \forall t, m, i, j\right. \\
& t_{i}^{\text {start }} \leq t \leq t_{i}^{\text {end }}, \quad \forall i=\text { O or } D
\end{aligned}
$$



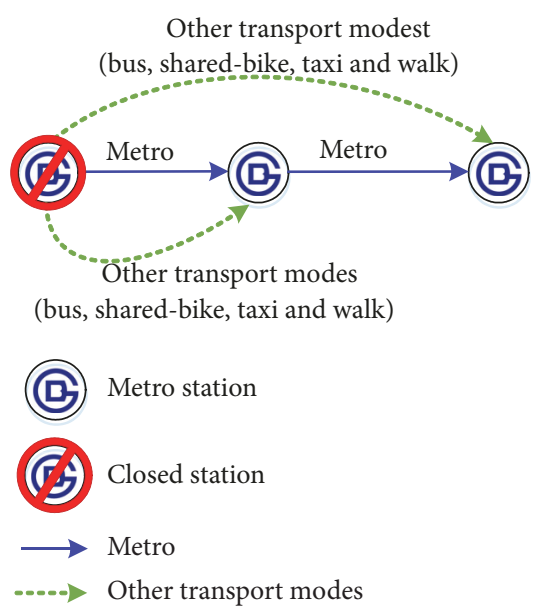

(a)
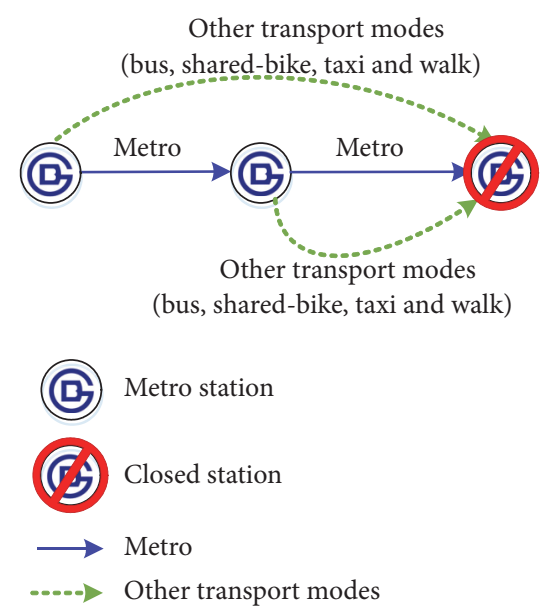

(b)

Figure 3: The two station disruption situations and likely choice behavior for a passenger. (a) shows the situation where the passenger's origin station is closed. (b) shows the situation that the passenger's destination station is closed.

Objective function (11) minimizes the generalized travel cost. Equations (12)-(14) indicate the basic constraints of the station disruption situation for a particular passenger, as illustrated in Figure 3. Constraint (12) indicates the generalized travel cost of making a new travel decision if the passenger's origin station is closed. Constraint (13) denotes the generalized travel cost of making a new travel decision if the passenger's destination station is closed. Constraint (14) indicates that only one case occurs for a particular passenger, which means that either the origin station $\left(\gamma_{O}^{p}=1, \gamma_{D}^{p}=\right.$ 0 , i.e., $b=\mathrm{O})$ or the destination station $\left(\gamma_{O}^{p}=0, \gamma_{D}^{p}=\right.$ 1 , i.e., $b=D)$ is closed.

Equations (15)-(16) denote the limits of the decision variables. Equation (15) denotes that another transport mode will bridge the closed origin station and the new departure station, or the new destination station and the closed destination station except the metro. Equation (16) denotes that an alternative station will be chosen to replace the closed one.

Equation (17) indicates the generalized cost of taking transport mode $m$ from station $i$ to station $j$ at time $t$ considering the travel time, price, and comfort penalty. $W$ is defined as a very large constant. When $x_{i}(\mathrm{t})=0$, it means that the generalized travel cost will be very large if the passenger $p$ still chooses the bridging-bus as his alternative transport mode. It also indicates that only when bridgingbuses are operated between stations and the closed station $b$, the bridging-buses can be available for passengers $\left(x_{i}(\mathrm{t})=1\right)$. It can be seen that the passenger's travel behavior is affected by the upper decision variables of controlling measures.

Equation (18) denotes that the time interval for a particular passenger to make a decision is only within the period of the station disruption. In other words, the model is applicable when the affected passenger has just been faced with the station disruption.
Three-Layer Discrete Choice Behavior Model for Alternative Travel Strategies. In a previous section, a behavior optimization model is proposed to generate an optimal travel strategy with a minimum generalized travel cost for the case of a station disruption. But, in an actual disruption scenario, the passengers' travel experience partially or completely turns into failure, and the necessary information for decisionmaking is usually lacking. Consequently, passengers cannot make a rational or optimal travel decision. Therefore, the randomness of passenger travel behavior should be considered in the newly proposed model.

Based on the stochastic utility theory, a three-layer nested-logit model for passengers' choice behavior under station disruption is constructed, which is illustrated in Figure 4.

In the proposed three-layer nested-logit model, the first layer is to make a trip decision among quitting the metro journey (labeled as the event $y_{1}$ ), waiting for the recovery of station service (labeled as event $y_{2}$ ) and taking an alternative metro station (labeled as event $y_{3}$ ); the second layer is to make travel decisions among the alternative stations set for the passengers taking an alternative metro station, or choices among various transport modes to continue the journey; the third layer is to make choices among available transportation modes to bridge the new alternative metro station and the planned origin or destination station. The multimodal transport resources contain bridging-bus, taxi, shared bicycle, and walking, which are labeled as $m 1, m 2, m 3$, and $m 4$, respectively. Accordingly, the general cost of each behavior is $c_{1}, c_{2}$, and $c_{3}$, respectively, for events $y_{1}, y_{2}$, and $y_{3}$. Take a passenger $p \in P_{O, D}^{t}$ as an example, and we can have these remarks.

(a) For the passenger who gives up the metro journey, the choice behavior is that the passenger will take the planned destination as his new departure station in the situation of 


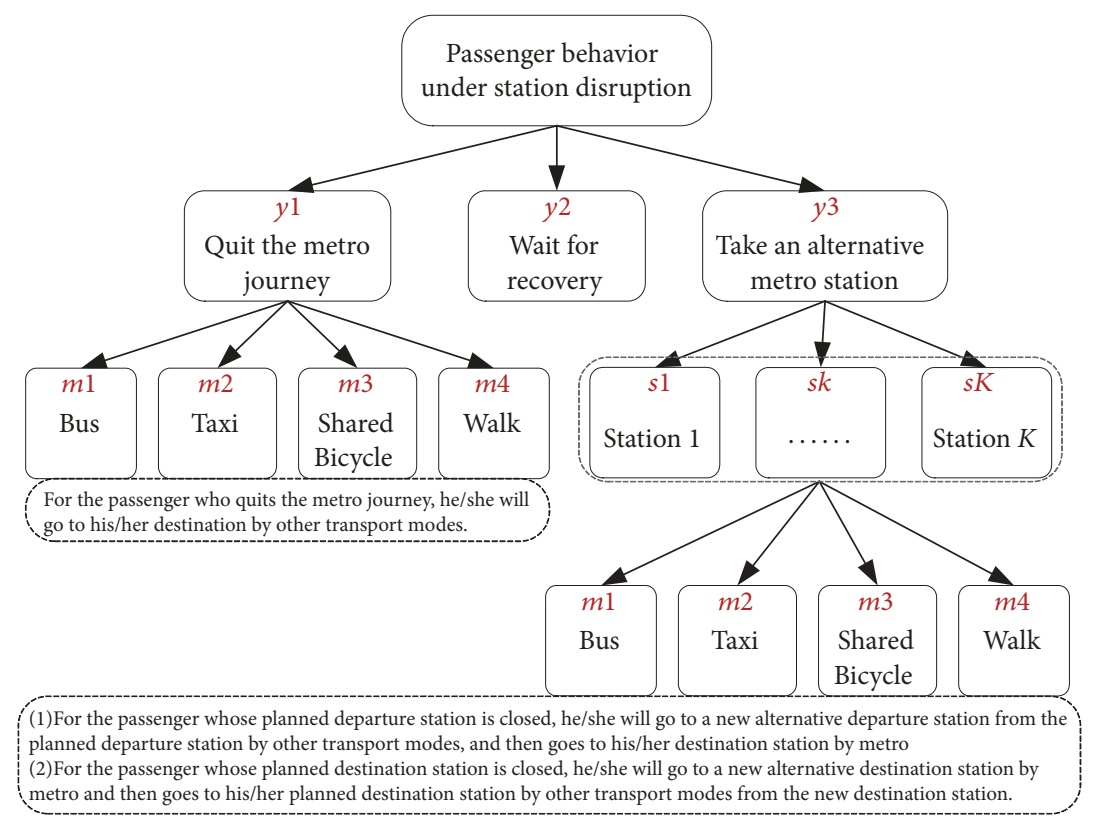

FIGURE 4: The nested-logit model for passenger behavioral choices under station disruption.

$\gamma_{O}^{p}=0$ and $\gamma_{D}^{p}=1$, or take the planned origin station as his new destination in the situation of $\gamma_{O}^{p}=1$ and $\gamma_{D}^{p}=0$. Then, the probability of choosing the model $m$ can be calculated as

$$
P\left(m \mid y_{1}\right)=\frac{\exp \left(-\theta c_{O D}^{m, t} / \min _{l \in M^{\prime}}\left\{c_{O D}^{l, t}\right\}+b\right)}{\sum_{m \in M^{\prime}} \exp \left(-\theta c_{O D}^{m, t} / \min _{l \in M^{\prime}}\left\{c_{O D}^{l, t}\right\}+b\right)}
$$

$\forall m \in M^{\prime}$

So $c_{1}$ can be calculated based on $(12) \sim(14)$ :

$$
c_{1}=\sum_{m \in M^{\prime}} P\left(m \mid y_{1}\right) c_{O D}^{m, t}
$$

(b) For the passenger who waits for the recovery of station service, the choice behavior is that the passenger will take the planned origin station as his new departure station. Once the destination is closed, it is hard to decide which station should be chosen to wait at. So waiting for the recovery of station service can be one option only in the case that one's planned departure station is closed. So $c_{2}$ can be calculated based on (12) (14):

$$
c_{2}=c_{O D}^{\text {rail }}
$$

(c) For the passenger who takes an alternative metro station, the choice behavior is that the passenger will take an alternative station as his new departure station or destination station. If the station $k$ is selected, the generalized travel cost of the passenger choosing the transport mode $m$ can be calculated as

$$
c_{3}^{m, k, t}= \begin{cases}c_{O, k}^{m}+c_{k, D}^{\text {metro }}, & \text { if } \mathrm{O} \text { is closed } \\ c_{\mathrm{O}, k}^{\text {metro }}+c_{k, D}^{m}, & \text { if } D \text { is closed }\end{cases}
$$

Firstly, in this situation, the probability of choosing the model $m$ can be calculated as

$$
\begin{aligned}
P( & \left.m \mid k, y_{3}\right) \\
& =\frac{\exp \left(-\theta c_{3}^{m, k, t} / \min _{l \in M^{\prime}}\left\{c_{3}^{l, k, t}\right\}+b\right)}{\sum_{r \in M^{\prime}} \exp \left(-\theta c_{3}^{r, k, t} / \min _{l \in M^{\prime}}\left\{c_{3}^{l, k, t}\right\}+b\right)}
\end{aligned}
$$

We label $c_{3}^{k}$ as the generalized travel cost of the passenger $p$ who chooses the station $k$ as his new alternative departure or destination station:

$$
c_{3}^{k}=\sum_{m \in M^{\prime}} P\left(m \mid k, y_{3}\right) c_{3}^{m, k, t}
$$

Then, the probability of choosing the station $k$ can be calculated as

$$
\begin{aligned}
& P\left(k \mid y_{3}\right)=\frac{\exp \left(-\theta c_{2}^{k} / \min _{l=1 \ldots K}\left\{c_{3}^{l}\right\}+b_{1}\right)}{\sum_{i}^{K} \exp \left(-\theta c_{3}^{i} / \min _{l=1 \ldots K}\left\{c_{3}^{l}\right\}+b\right)} \\
& \forall k=1 \ldots K \\
& c_{3}=\sum_{k} P\left(k \mid x_{2}\right) c_{2}^{k}
\end{aligned}
$$

The probability of each passenger behavior decision is

$$
\begin{aligned}
P\left(y_{j}\right)=\frac{\exp \left(-\theta c_{j} / \min _{l=1,2,3}\left\{c_{l}\right\}+b_{2}\right)}{\sum_{i=1}^{3} \exp \left(-\theta c_{i} / \min _{l=1,2,3}\left\{c_{l}\right\}+b\right)} & \\
& \forall j=1,2,3
\end{aligned}
$$

The joint probability of passenger choosing a station $k$ as an alternative station can be calculated:

$$
P\left(k, y_{3}\right)=P\left(k \mid y_{3}\right) P\left(y_{3}\right)
$$




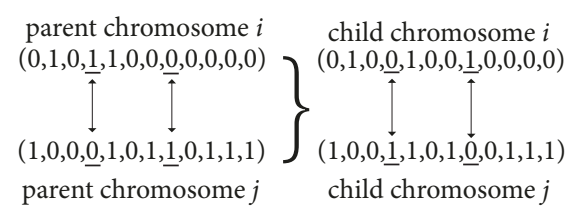

FIgURE 5: The crossover operator.

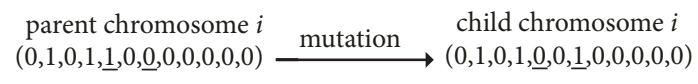

FIgURE 6: The mutation operator.

Furthermore, the joint probability of choosing transport mode $m$ can be

$$
\begin{gathered}
P\left(m, y_{1}\right)=P\left(m \mid y_{1}\right) P\left(y_{1}\right) \\
P\left(m, k, y_{3}\right)=P\left(m \mid k, y_{3}\right) P\left(k, y_{3}\right)
\end{gathered}
$$

\subsection{An Integrated Solution Algorithm}

2.4.1. Genetic Algorithm. The decision variable in the upper model is $x_{i}(t)$, indicating whether the bridging-bus should be operated between the closed station and the station $i \in S$ at the time interval $t .1$ denotes "YES" and 0 stands for "NO." Therefore, in the genetic algorithm, the traditional binary coding method can be used to design a feasible solution as the corresponding gene sequence or chromosome. A chromosome is represented by a vector as indicated in (31). Besides, the population is represented by $H$ in (32).

$$
\begin{aligned}
H_{l} & =\left({ }_{l} x_{0}, \ldots,{ }_{l} x_{i}, \ldots,{ }_{l} x_{n}\right) \\
H & =\left\{H_{1}, H_{2}, \ldots, H_{L}\right\}
\end{aligned}
$$

Generally, the genetic algorithm consists of three important operators: the crossover operator, the mutation operator, and the selection operator. We assume that the chromosome intersects with a certain probability and randomly selects the intersection with a certain crossover probability $\xi$. In the algorithm, the crossover method is given as shown in Figure 5. Since the chromosome is composed of a specific gene fragment which cannot be divided only the same position of the gene fragment will be crossed.

The mutation operator can help avoid local optimal solutions. In this paper, we assume that each chromosome will be a certain probability of mutation operation $\zeta$ and randomly select the mutation position, as shown in Figure 6 .

The selection operator is to ensure the best individuals can survive in the next generation. And it is designed based on the fitness function, defined as $f(n)=1 / Z$, where $Z$ is defined by formula (4). The more the fitness is, the more likely the best individual can survive. We adopt the Roulette method to determine whether each individual can survive in the next generation or not.

2.4.2. Solution Algorithm of Passenger Behavior Model. The general simulation procedure can be attributed to Yin et al.
[1]. A new behavior model solver in the simulation algorithm is developed for the three-layer discrete choice behavior model, which is summarized as shown in Algorithm 1:

2.4.3. The General Solution Algorithm. In this paper, a genetic algorithm and a simulation based method are integrated to solve the proposed model. The genetic algorithm is used to solve the binary integer programing model and the simulation procedure is to generate different types of the passenger flow demand. And the basic simulation procedure is attributed to Yin et al. [1]. The general algorithm framework for solving the problem of station disruption controlling optimization is described as follows.

Step 1 (initialize). Initialize the parameters and set the maximum number of iterations to $G$, and the population size is $L$. Initialize the disruption's time duration as $[1, n]$. Set the current time interval $t=1$.

Step 2. Set an initial set of bus-bridging scheme or initial population. Set the current generation $g=0$.

Step 3. Solve the lower model. For a particular bus-bridging scheme, the simulation model and algorithm are called to capture the passenger travel behavior and each passengers' travel time. And calculate Z.

Step 4. Solve the upper model. According to each passengers' travel time, the fitness of each chromosome in the initial population can be calculated and through the following operations, to generate the upper optimization of the busbridging scheme strategy $x_{i}$.

Step 4.1. Mutation operation: using the mutation operator to create a new chromosome.

Step 4.2. Cross operation: cross the operation on the $L$ chromosome.

Step 4.3. Selection operation: using the selection operator to ensure that the optimal individual genetic to the next generation.

Step 5. Verify the end condition 1 . If the number of iterations is greater than $G$, then the optimal solution of the model will be obtained; otherwise, $g=g+1$, and the iteration will return to Step 3.

Step 6. Verify the end condition 2 . If $t>n$, end; otherwise, make $t=t+1$, and return to Step 2 .

\section{Numerical Example}

In this section, we present the application of suggested model and the solution algorithm to an example urban metro network based on real and virtual data. Figure 7 illustrates the example station disruption scenario, which contains 2 directional lines and 2 transfer stations extracted from Beijing Subway network, which is one of the busiest metro systems [23]. Figure 8 illustrates the practical train timetables. We 
Initialization: initialize the corresponding parameters of discrete choice behavior model, and $K$.

Procedure:

(1) if $\gamma_{O}^{p}==1$ then

(2) Define a temporary set $L_{i s t} k$;

(3) for $s \in S$ do

(4) if $s==O$ then

(5) Calculate the $c_{2}$ based on the Eq. (21)

(6) else if $s==D$ then

(7) Calculate the $c_{1}$ based on the Eqs. (19) $\sim(20)$.

(8) else

(9) Calculate the $c_{3}^{s}$ based on the Eqs. (22) (24).

(10) if the number of elements in List_k is not exceeding $K$, add the $c_{3}^{s}$ into the set List_$_{-} K$ and sort the elements from smallest to largest;

(11) if the number of elements in List_k equals $K$, and the last value of List_k is larger than $c_{3}^{s}$, remove the last value of $L i s t_{-} k$ and add $c_{3}^{s}$ to the $L_{i s t} k$.

(12) end if.

end for.

(13) Calculate the $c_{3}$ based on the $c_{3}^{s} \in$ List $k$ using the Eqs. (25) (26).

(14) Then, calculate $P\left(y_{j}\right)$ based on Eqs. (27).

(15) Calculate the joint probability $P\left(m, y_{1}\right), P\left(k, y_{3}\right), P\left(m, k, y_{3}\right)$ based on Eqs. (28) (30).

(16) Using the Roulette method to generate an alternative travel decision:

(17) (1) Construct a cumulative probability set $R$ based on $P\left(m, y_{1}\right), P\left(y_{2}\right), P\left(m, k, y_{3}\right)$, where $m=1,2,3,4$, and $k=1,2, \ldots, K$.

$$
\begin{aligned}
& R[m-1]=\sum_{l=1}^{m} P\left(l, y_{1}\right), m=1,2,3,4 \\
& R[4 k+m-1]=\sum_{l=1}^{4} P\left(l, y_{1}\right)+\sum_{u=1}^{k-1} \sum_{v=1}^{4} P\left(v, \mathrm{u}, y_{3}\right)+\sum_{v=1}^{m} P\left(v, k, y_{3}\right), m=1,2,3,4, k=1,2, \ldots, K .
\end{aligned}
$$$$
R[4(K+1)]=\sum_{l=1}^{4} P\left(l, y_{1}\right)+\sum_{u=1}^{K} \sum_{v=1}^{4} P\left(v, u, y_{3}\right)+P\left(y_{2}\right)=1
$$

(2) Generate a random variable $r$.

(3) Determine the behavior choices:

if $r \leq R[0]$, passenger will give up the metro journey and take a bus instead to the destination and $v_{O}^{\text {bus }, t}++$; if $R[m-1]<r \leq R[m]$, passenger will give up the metro journey and take the transport mode $m$ instead to the destination, $v_{O}^{m, t}++$; if $R[4 k+m-1]<r \leq R[4 k+m]$, passenger will take an alternative station $k$ as his new origin station of metro journey, and he will take the transport mode $m$ to the new origin station from the planned origin station, the entry time at new origin is the original entry time added by the time consumption of the transport mode $m, v_{O}^{m, t}++$;

if $R[4 K+3]<r \leq 1$, passenger will wait at the planned origin station.

(28) Define a temporary set List_k , and we set the $O$ as the passengers' current station at the current time. If passenger $p$ is now in train, we set the $O$ as the next station the train will stop at.

(29) Judge whether the travel time from the current station to the destination is larger than the closure duration? if yes, the passenger $p$ need not to change his travel behavior. If not, go on executing the following part.

(30) for $s \in S$ do

(31) if $s==O$ then

(32) Calculate the $c_{1}$ based on the Eqs. (19) (20).

(33) else if $s==D$ then

(34) Set $c_{2}=W$, where $W$ is a very big constant.

(35) else

(36) Calculate the $c_{3}^{s}$ based on the Eqs. (22) (24).

(37) if the number of elements in List_ $k$ is not exceeding $K$, add the $c_{3}^{s}$ into the set List_K and sort the elements from smallest to largest;

(38) if the number of elements in List_k equals $K$, and the last value of $L i s t_{-} k$ is larger than $c_{3}^{s}$, remove the last value of List_k and add $c_{3}^{s}$ to the List_k.

(39) end if.

(40) end for.

(41) Calculate the $c_{3}$ based on the $c_{3}^{s} \in$ List $\_$using the Eqs. (25) (26).

(42) Then, calculate $P\left(y_{j}\right)$ based on Eq. (27).

(43) Calculate the joint probability $P\left(m, y_{1}\right), P\left(k, y_{3}\right), P\left(m, k, y_{3}\right)$ based on Eqs. (28) (30). 
(44) Using the Roulette method to generate an alternative travel decision:

(45) (1) Construct a cumulative probability set $R$ based on $P\left(m, y_{1}\right), P\left(y_{2}\right), P\left(m, k, y_{3}\right)$, where $m=1,2,3,4$, and $k=1,2, \ldots, K$.

(46) $\quad R[m-1]=\sum_{l=1}^{m} P\left(l, y_{1}\right), m=1,2,3,4$

(47) $\quad R[4 k+m-1]=\sum_{l=1}^{4} P\left(l, y_{1}\right)+\sum_{u=1}^{k-1} \sum_{v=1}^{4} P\left(v, \mathrm{u}, y_{3}\right)+\sum_{v=1}^{m} P\left(v, k, y_{3}\right), m=1,2,3,4, k=1,2, \ldots, K$.

(48) (2) Generate a random variable $r$.

(49) (3) Determine the behavior choices:

(50) if $r \leq R[0]$, passenger will give up the metro journey and take a bus instead to the destination from current station, $v_{O}^{\text {bus }, t}++$;

(51) if $R[m-1]<r \leq R[m]$, passenger will give up the metro journey and take the transport mode $m$ instead to the destination from current station, $v_{O}^{m, t}++$;

(52) if $R[4 k+m-1]<r \leq R[4 k+m]$, passenger will take an alternative station $k$ as his new destination station of metro journey, and he will take the transport mode $m$ to the planned destination end if. station from the new destination station, $v_{k}^{m, t}++$;

Algorithm 1: Solution algorithm of the passenger behavior model.

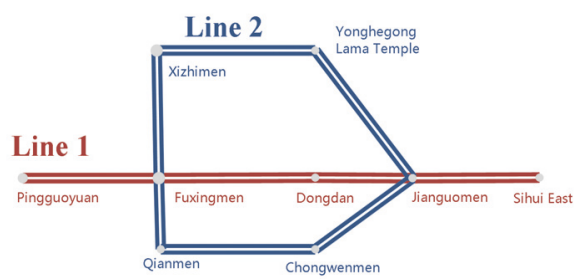

Figure 7: An example of a metro network extracted from Beijing metro network.

assume that the Xizhimen station is closed. The cost of taking alternative transport modes is given in Table 1 .

In order to compute and test the model more easily, we assume that each OD volume shares the same distribution, as shown in Figure 9. Different values of $V$ can be investigated for different scales of passenger demand. And other values of main parameters are defined in Table 2 for simplification.

$$
f^{o d}(t)= \begin{cases}\frac{V}{12} \times t, & t \in[0,12) \\ -\frac{V}{16} \times t+\frac{7 V}{4}, & t \in[12,28)\end{cases}
$$

where $t$ denotes the $t$ th time interval of 15 minutes and $V$ indicates the maximum passenger demand of each two different stations per time interval, indicated in Figure 9.

\subsection{Passenger Flow Demand Analysis under a Station Disruption}

\subsubsection{Analysis of Individual Behavioral Choices}

Passengers' Behavior Choices under Different the Closure Duration. For a particular OD from the Xizhimen station to the Pingguoyuan station, Figure 10 shows that, with increasing closure duration, the passengers will more likely alter their planned origin stations or give up their metro journeys, and fewer passengers will wait for its recovery of station service.
Alternatives of Transport Modes. Figure 11 shows the passengers' choices in multimodal transports. With increasing the bridging distance up to $6 \mathrm{~km}$, more passengers prefer to choose the taxi as their alternative transport mode.

Alternatives of Origin Stations. Figure 12 shows the top stations that passengers prefer to choose as their alternative origins when their planned origin station Xizhimen station is closed for different durations. The Fuxingmen, which is near the Xizhimen station, is always chosen by most passengers. The result also shows that fewer passengers will wait at the closed station and more passengers will give up their planned origin stations to nearby stations if the closure duration becomes larger.

\subsubsection{Passenger Flow Demand at Affected Stations}

Passenger Flow Dynamics at the Closed Station. In a 60 minutes' station disruption, the number of entries at the Xizhimen station is changing over time, as is shown in Figure 13. It also shows that when the disruption comes to an end, the passengers waiting at closed station will gradually enter the closed station, which leads to more entries than normal. And, consequently, it takes longer than the closure duration itself for the entries to recover to a normal level, as shown in Figure 13.

Passenger Flow Dynamics at the Nearby Stations. The disruption of Xizhimen station results in a significant increase in the number of entries at the nearby stations, especially at the Fuxingmen station, as is shown in Figure 14.

\subsection{Optimization Results.}

Optimization Results under Different Disruption Durations. As is indicated in Figure 15, a set of disruption scenarios are numerically computed for a determined OD volume of $V=240$, and the duration of station disruption ranges from 0 to 120 minutes. The results show that when the duration 


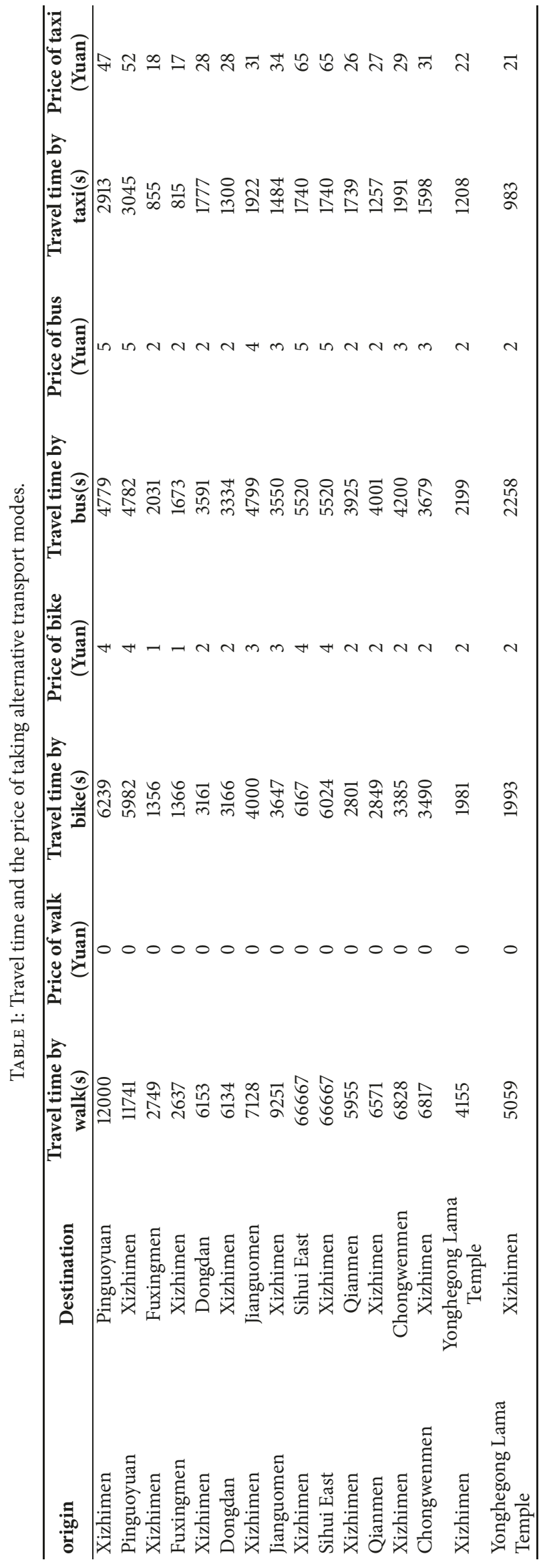



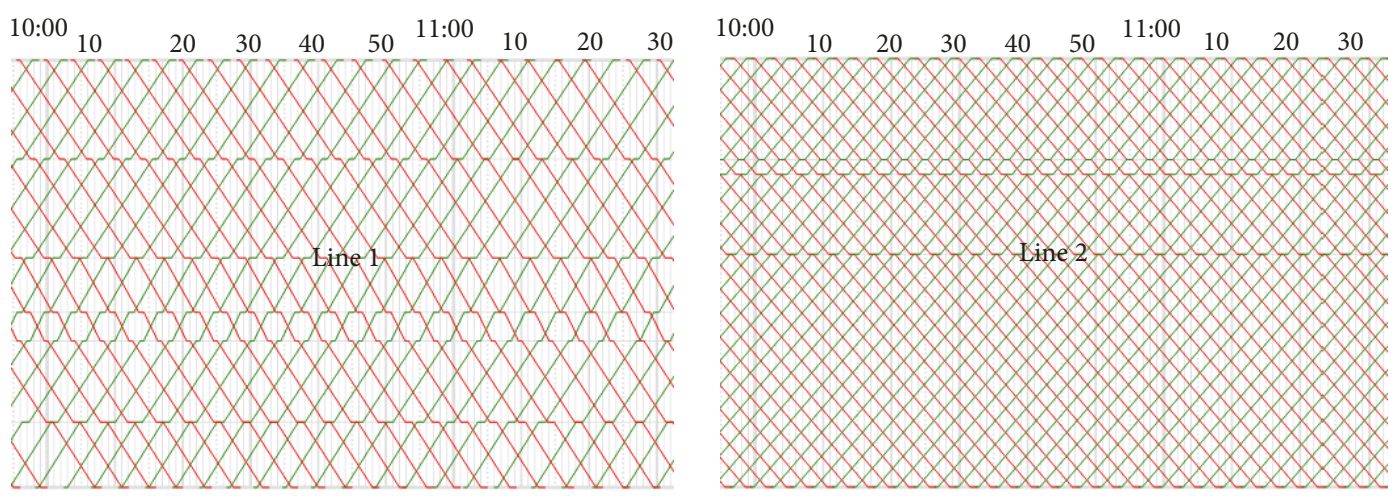

Figure 8: The partial train diagram of each metro line. The horizontal axis denotes the time. For example, the "10" after the "10:00" indicates the time of 10:10, and so on.

TABLE 2: Values of some parameters in the numerical study.

\begin{tabular}{llc}
\hline Parameters & Definitions & Values \\
\hline$\mu$ & the parameter of transferring the buses' running distance to the standardized operating cost & 100 \\
$\gamma$ & the parameter of transferring the passengers' travel time to the standardized travel cost & 1 \\
$\theta$ & the magnification parameter of the generalized cost in the logit model & 3 \\
$b$ & a random constant associated with the generalized cost in the logit model & 0 \\
$\alpha_{m}$ & the value of the travel time of urban transport mode $m$, which can be found in local studies in China \\
$\beta_{\text {walk }}$ & the comfort penalty parameter of walk \\
$\beta_{b i k e}$ & the comfort penalty parameter of bike \\
$\beta_{\text {bus }}$ & the comfort penalty parameter of bus \\
$\beta_{\text {taxi }}$ & the comfort penalty parameter of taxi \\
$\xi$ & crossover probability in genetic algorithm \\
$\zeta$ & mutation probability in genetic algorithm & 30 \\
$G$ & the upper limit of the number of iterations in the genetic algorithm \\
$L$ & population size in genetic algorithm & 1.8 \\
\hline
\end{tabular}

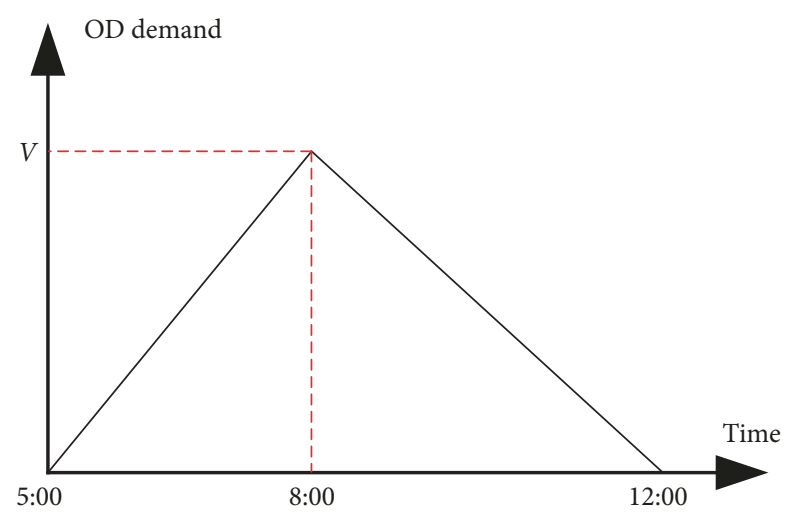

FIGURE 9: The OD passenger flow demand over time.

of station disruption becomes larger, the total travel time will be more significantly reduced if the bridging-buses are operated. The improvement can reach up to $49.38 \%$ under a station disruption of 120 minutes. That is because when

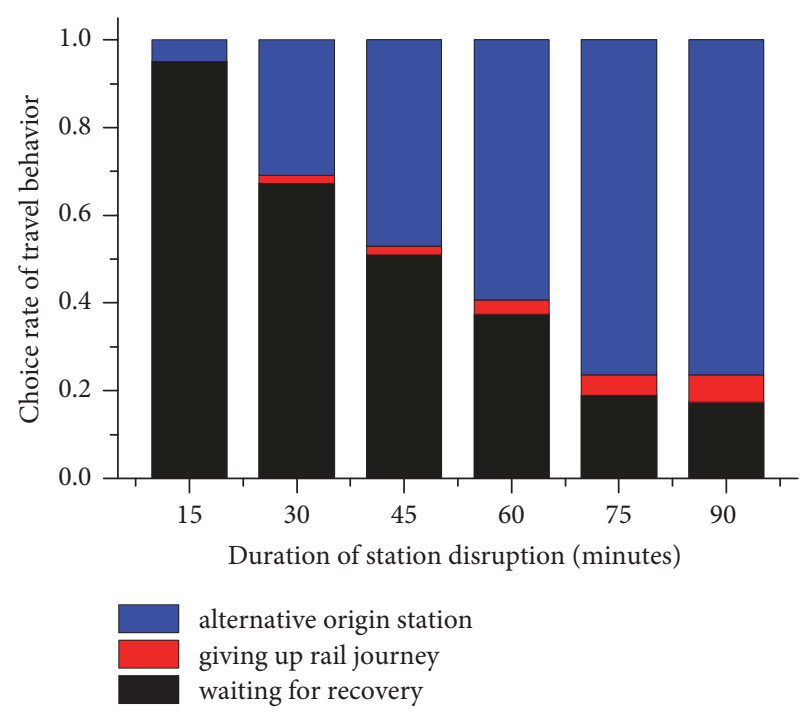

FIGURE 10: The relationship between the duration of station disruption and the passenger behavioral choices. 


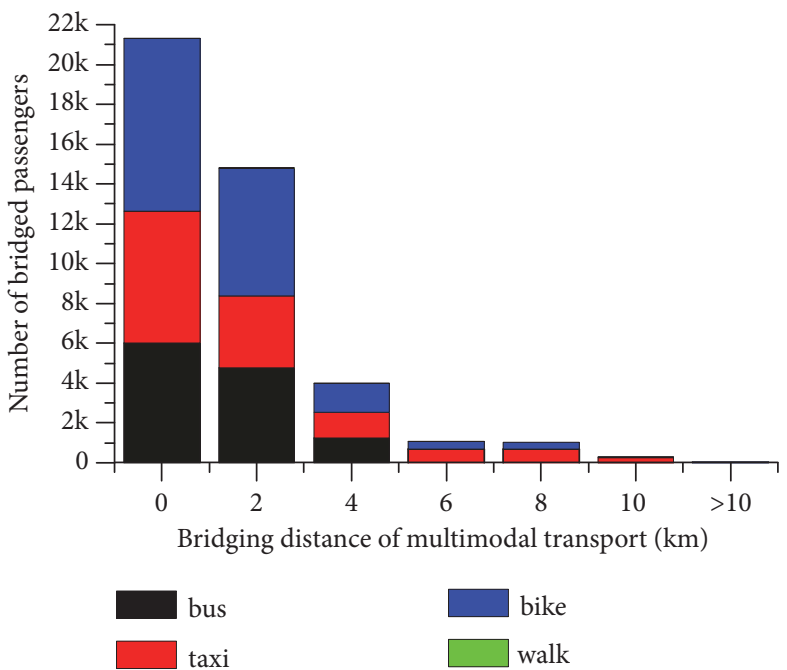

(a)

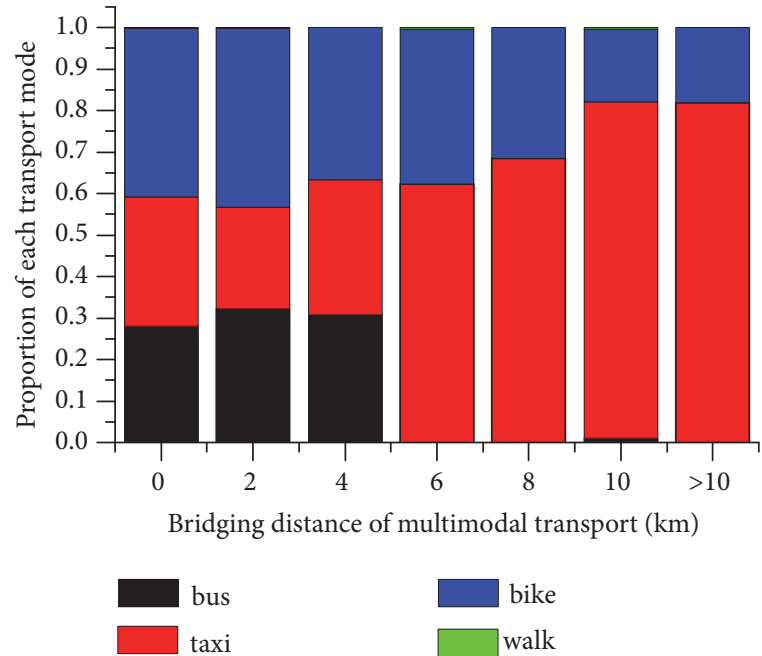

(b)

FIGURE 11: Alternatives of transport modes. (a) shows the numbers of bridging passengers are decreasing sharply with the increases in bridging distance; (b) shows the proportion of passengers choosing the different transport mode with the changes of bridging distance.

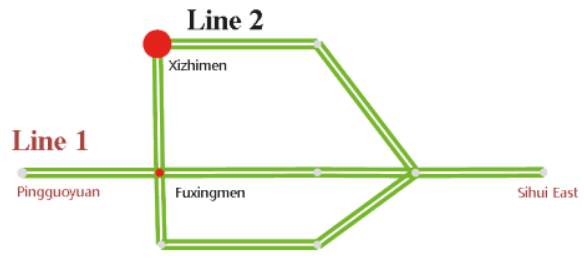

(a) $15 \mathrm{~min}$

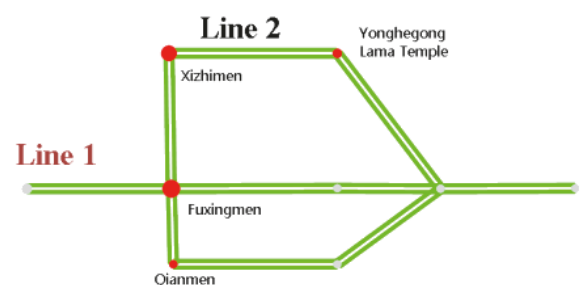

(c) $45 \mathrm{~min}$

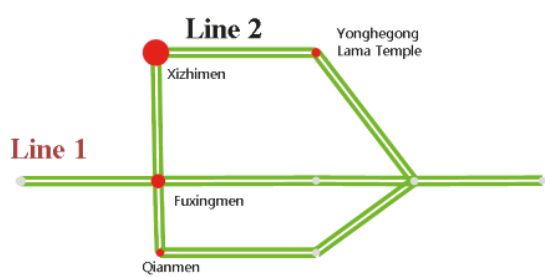

(b) $30 \mathrm{~min}$

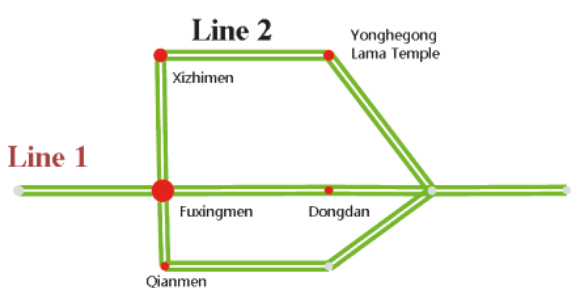

(d) $60 \mathrm{~min}$

Figure 12: The replaceable departure stations passengers prefer to choose when Xizhimen is closed for different closure durations. The red circles represent the alternative origin stations, including the closed station. The size of each circle represents the number of passengers who choose that station, and a larger radius indicates relatively more passengers. (a) shows the duration of 15 minutes; (b) shows the duration of 30 minutes; (c) shows the duration of 45 minutes; (d) shows the duration of 60 minutes.

the station is closed for a longer time, more passengers will choose the bridging-buses or other transport modes instead of waiting for recovery of the station service, which leads to a better controlling effect. It also indicates that, in the minor disruption scenario, there is no need to operate the bridgingbuses.

The blue line in Figure 15 also shows that our developed solution algorithm is time-expensive. It costs up to 305 minutes in an only 120 minutes' station disruption scenario in the small numerical study, which seems not to be efficient enough for large-scale network cases or on-line emergent situations. That is because our proposed model must calculate all behavioral responses to the station disruption for every affected passenger in each iteration in the solution algorithm.

Optimization Results under Different Passenger Flow. As is indicated in Figure 16, another set of disruption scenarios are numerically computed for a disruption duration of 60 minutes, and the OD volume of $V$ ranges from 60 to 420 . The results show that, with increasing the passenger flow demand, more total travel time will be significantly reduced if the bridging-buses are operated. 


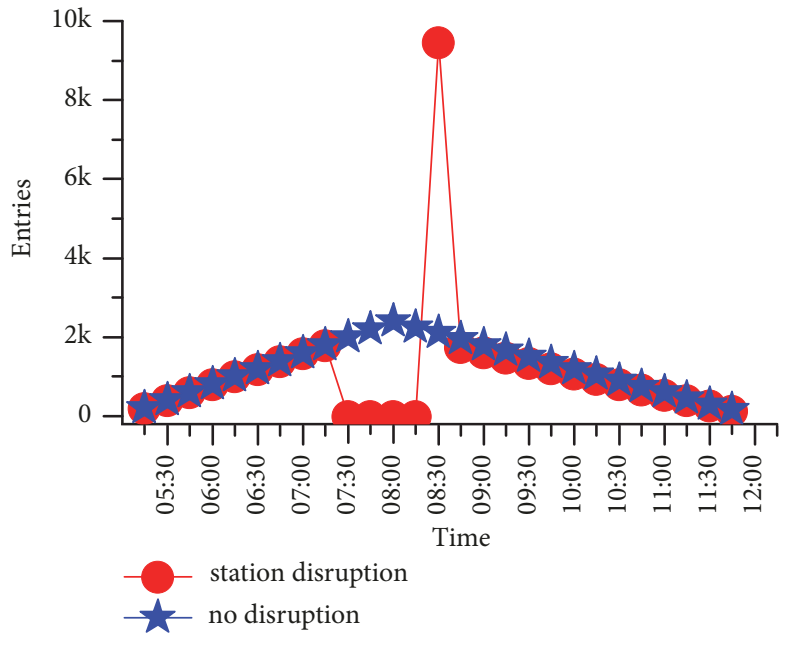

(a)

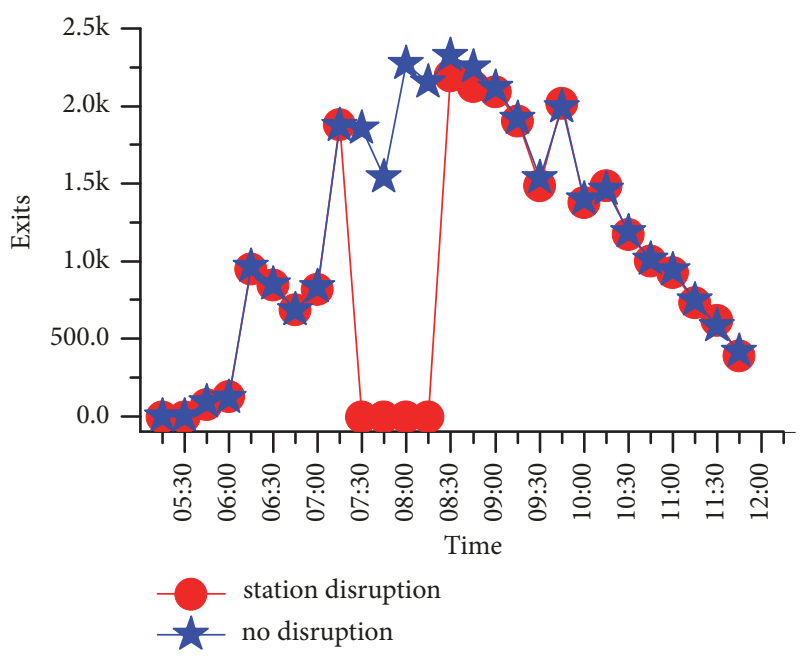

(b)

Figure 13: The number of entries and exits at the closed station Xizhimen. (a) shows entries of Xizhimen over time; (b) shows the exits of Xizhimen over time.

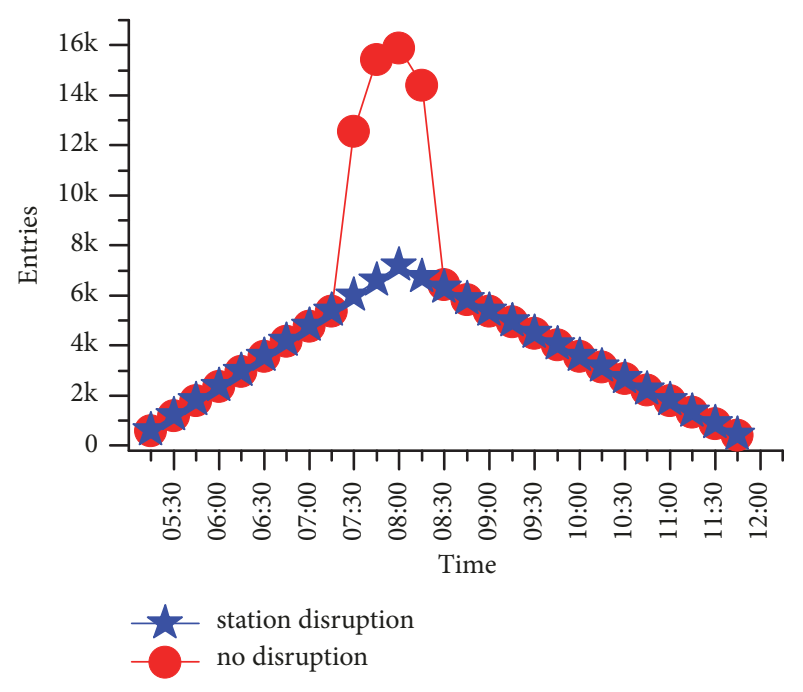

(a)

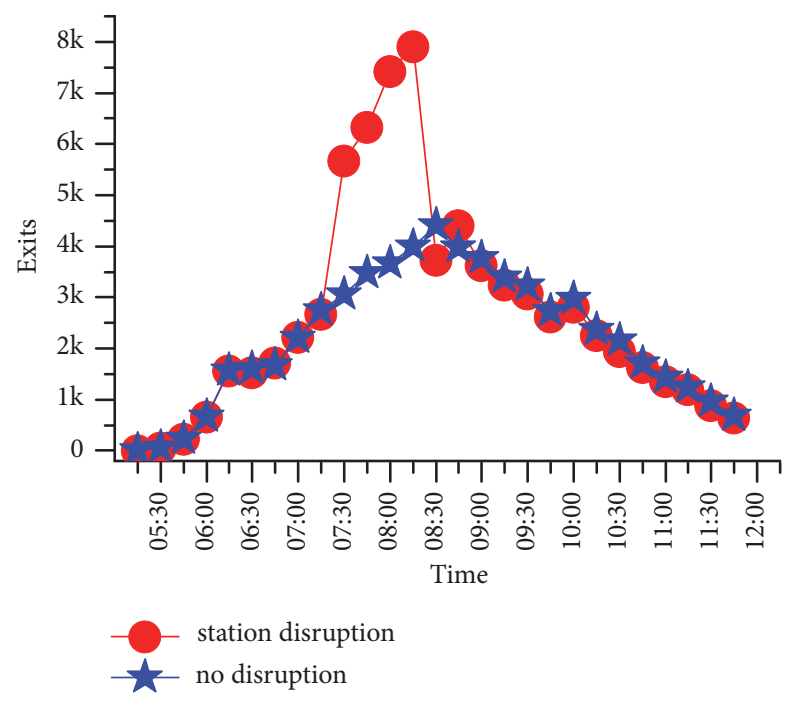

(b)

FIGURE 14: The number of entries and exits at the affected station-Fuxingmen. (a) shows entries of Fuxingmen over time; (b) shows the exits of Fuxingmen over time.

\section{Conclusion}

An integrated optimization approach based on behavior model is proposed to manage and control the disruption crisis by providing additional bus-bridging services with the objective of minimizing the total travel time of affected passengers and the operating cost of bridging-buses. Among them, a three-layer discrete choice behavior model is proposed to analyze the passenger flow demand under station disruption. Furthermore, the multimodal transport modes, including metro, bridging-bus, shared-bike, and taxi, are considered as passengers' alternative choices in face of the station disruption. A numerical study based on Beijing metro network is conducted finally. Some conclusions can be drawn as follows.

(a) The effects of a station disruption with different durations on passenger flow demand are analyzed, and the results show that, with increasing closure duration, more passengers will alter their planned origin stations or quit their metro journey, and fewer passengers will wait at the closed station for its recovery. And, with increasing the bridging distance up to $6 \mathrm{~km}$, more passengers prefer to choose the taxi as their alternative transport mode.

(b) The optimization results show that additional busbridging services can eliminate the station disruption impact significantly, especially with the increase of closure duration 


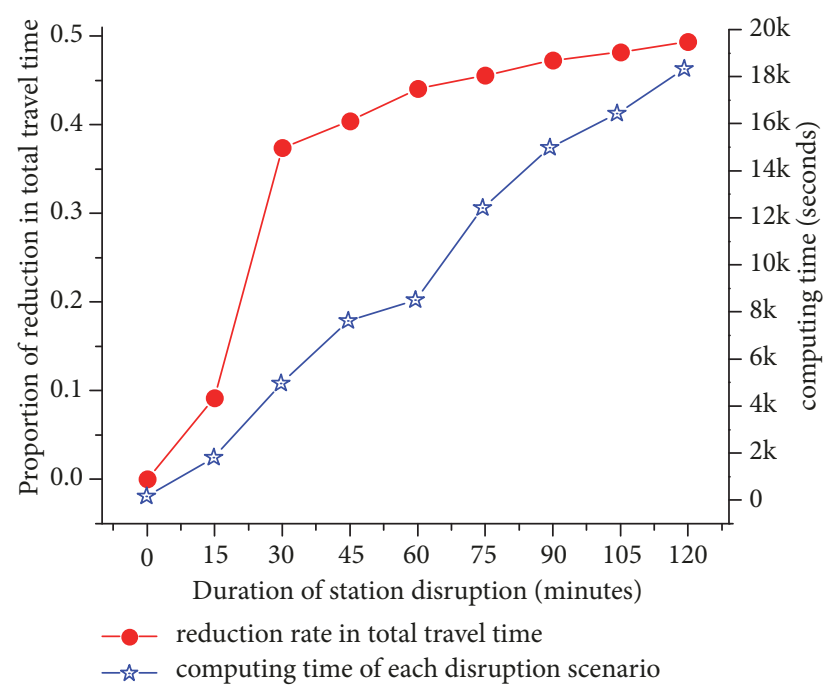

Figure 15: The optimization results under different disruption durations.

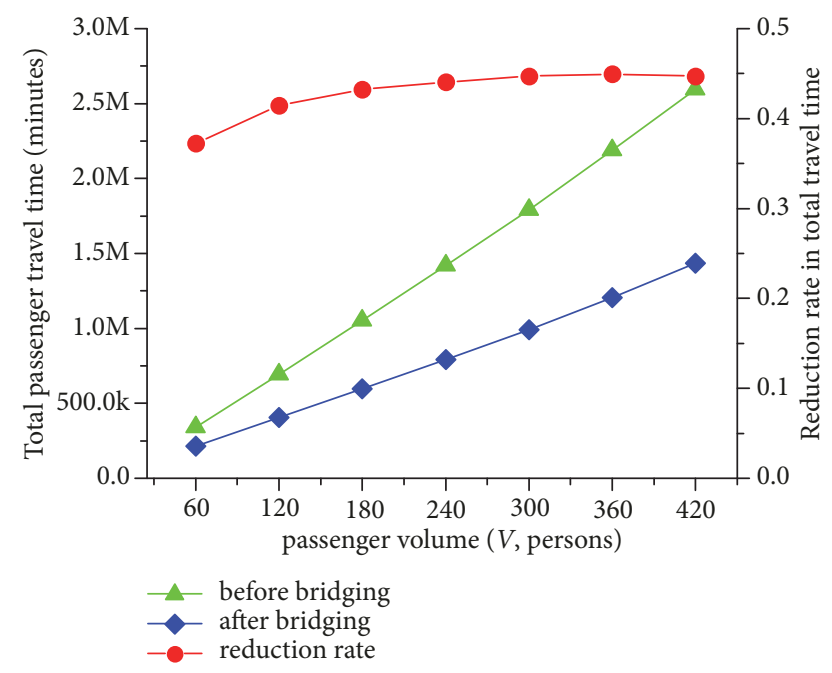

FIGURE 16: The optimization results under different passenger flow demand.

and the passenger flow demand. The improvement can reach up to $49.38 \%$ under a station disruption of 120 minutes and a determined OD volume of $V=240$ in our numerical study.

(c) Our developed solution algorithm is time-expensive and seems not to be efficient and applicable enough for largescale network cases or real-time emergent situations. A more efficient algorithm should be developed in our future studies.

\section{Data Availability}

All data generated and/or analyzed during the current study are available from the corresponding author on reasonable request.

\section{Conflicts of Interest}

The authors declare that they have no conflicts of interest.

\section{Acknowledgments}

This work was supported by NSFC (nos. 71771018, 71621001, 71701013, and 71601017), the Fundamental Research Funds for the Central Universities (no. 2018JBM074), Research Foundation of State Key Laboratory of Rail Traffic Control and Safety (no. RCS2018ZT004), and Technology Foundation of Beijing Jiaotong University (no. 2018RC010). The authors thank these agencies.

\section{References}

[1] H. Yin, B. Han, D. Li, J. Wu, and H. Sun, "Modeling and simulating passenger behavior for a station closure in a rail transit network," PLoS ONE, vol. 11, no. 12, Article ID e0167126, 2016.

[2] L. Cadarso, Á. Marín, and G. Maróti, "Recovery of disruptions in rapid transit networks," Transportation Research Part E: Logistics and Transportation Review, vol. 53, no. 1, pp. 15-33, 2013.

[3] X. He and H. X. Liu, "Modeling the day-to-day traffic evolution process after an unexpected network disruption," Transportation Research Part B: Methodological, vol. 46, no. 1, pp. 50-71, 2012.

[4] J. Jespersen-Groth, D. Potthoff, J. Clausen et al., "Disruption management in passenger railway transportation," in Robust and Online Large-Scale Optimization, R. K. Ahuja, R. H. Möhring, and C. D. Zaroliagis, Eds., pp. 399-421, Springer, Berlin, Germany, 2009.

[5] L. K. Nielsen, Rolling Stock Rescheduling in Passenger Railways: Applications in short-term planning and in disruption management, Erasmus University Rotterdam, 2011.

[6] B. Pender, G. Currie, A. Delbosc, and N. Shiwakoti, "Planning for the unplanned: An international review of current approaches to service disruption management of railways," in Proceedings of the 35th Australasian Transport Research Forum, ATRF 2012, aus, September 2012.

[7] R. Silva, S. M. Kang, and E. M. Airoldi, "Predicting traffic volumes and estimating the effects of shocks in massive transportation systems," Proceedings of the National Acadamy of Sciences of the United States of America, vol. 112, no. 18, pp. 56435648, 2015.

[8] Y. Chung, "A methodological approach for estimating temporal and spatial extent of delays caused by freeway accidents," IEEE Transactions on Intelligent Transportation Systems, vol. 13, no. 3, pp. 1454-1461, 2012.

[9] C. Liu, S. Zhang, H. Wu, and Q. Fu, "A Dynamic Spatiotemporal Analysis Model for Traffic Incident Influence Prediction on Urban Road Networks," ISPRS International Journal of GeoInformation, vol. 6, no. 11, p. 362, 2017.

[10] J. Wu, M. Liu, H. Sun, T. Li, Z. Gao, and D. Z. W. Wang, "Equity-based timetable synchronization optimization in urban subway network," Transportation Research Part C: Emerging Technologies, vol. 51, pp. 1-18, 2015.

[11] X. Guo, J. Wu, H. Sun, R. Liu, and Z. Gao, "Timetable coordination of first trains in urban railway network: a case study of Beijing," Applied Mathematical Modelling: Simulation and Computation for Engineering and Environmental Systems, vol. 40, no. 17-18, pp. 8048-8066, 2016.

[12] X. Guo, H. Sun, J. Wu, J. Jin, J. Zhou, and Z. Gao, "Multiperiodbased timetable optimization for metro transit networks," 
Transportation Research Part B: Methodological, vol. 96, pp. 4667, 2017.

[13] X. Yang, X. Li, B. Ning, and T. Tang, "An optimisation method for train scheduling with minimum energy consumption and travel time in metro rail systems," Transportmetrica B: Transport Dynamics, vol. 3, no. 2, pp. 79-98, 2015.

[14] J. Yang, J. G. Jin, J. Wu, and X. Jiang, "Optimizing Passenger Flow Control and Bus-Bridging Service for Commuting Metro Lines," Computer-Aided Civil and Infrastructure Engineering, vol. 32, no. 6, pp. 458-473, 2017.

[15] X. Yang, A. Chen, B. Ning, and T. Tang, "Bi-objective programming approach for solving the metro timetable optimization problem with dwell time uncertainty," Transportation Research Part E: Logistics and Transportation Review, vol. 97, pp. 22-37, 2017.

[16] X. Yang, A. Chen, J. Wu, Z. Gao, and T. Tang, "An energyefficient rescheduling approach under delay perturbations for metro systems," Transportmetrica B: Transport Dynamics, pp. 1$15,2018$.

[17] H. Yin, J. Wu, H. Sun, L. Kang, and R. Liu, "Optimizing last trains timetable in the urban rail network: social welfare and synchronization," Transportmetrica B: Transport Dynamics, pp. 1-25, 2018.

[18] J. Yin, Y. Wang, T. Tang, J. Xun, and S. Su, "Metro train rescheduling by adding backup trains under disrupted scenarios," Frontiers of Engineering Management, vol. 4, no. 4, p. 418, 2017.

[19] K. Kepaptsoglou and M. G. Karlaftis, "The bus bridging problem in metro operations: Conceptual framework, models and algorithms," Public Transport, vol. 1, no. 4, pp. 275-297, 2009.

[20] Y. Wang, J. Guo, G. Currie, A. Ceder, W. Dong, and B. Pender, "Bus bridging disruption in rail services with frustrated and impatient passengers," IEEE Transactions on Intelligent Transportation Systems, vol. 15, no. 5, pp. 2014-2023, 2014.

[21] J. G. Jin, K. M. Teo, and L. Sun, "Disruption response planning for an urban mass rapid transit network[C]," in Transportation Research Board (TRB) 92nd Annual Meeting, pp. 1-18, Transportation Research Board, Washington DC, USA, 2013.

[22] J. G. Jin, K. M. Teo, and A. R. Odoni, "Optimizing bus bridging services in response to disruptions of urban transit rail networks," Transportation Science, vol. 50, no. 3, pp. 790-804, 2016.

[23] K. Lu, B. Han, F. Lu, and Z. Wang, "Urban Rail Transit in China: Progress Report and Analysis (2008-2015)," Urban Rail Transit, vol. 2, no. 3-4, pp. 93-105, 2016. 


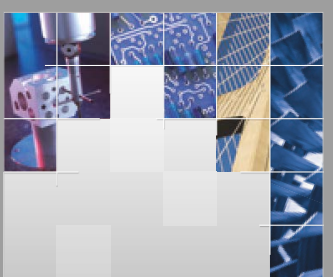

\section{Enfincering}
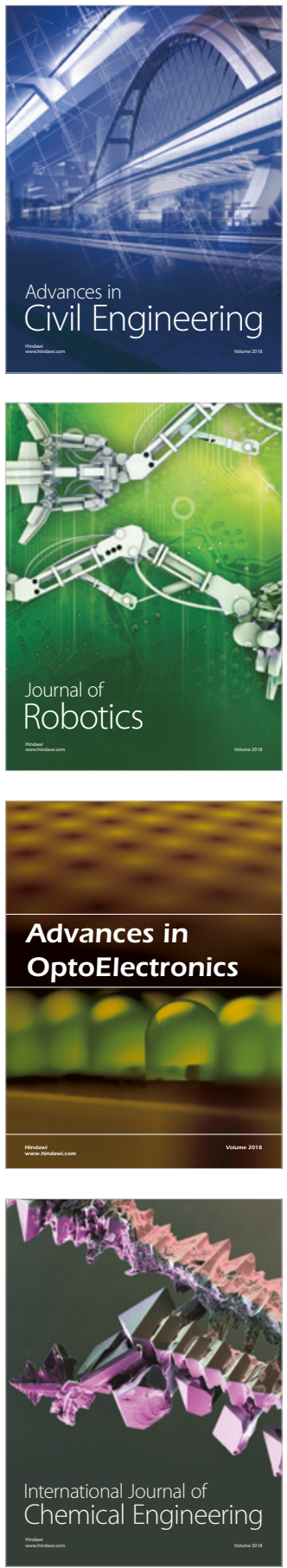

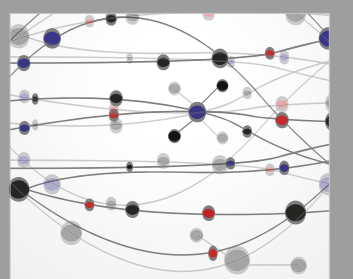

\section{Rotating \\ Machinery}

The Scientific World Journal

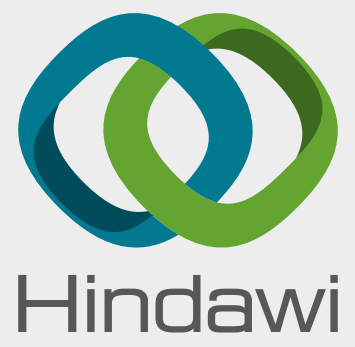

Submit your manuscripts at

www.hindawi.com
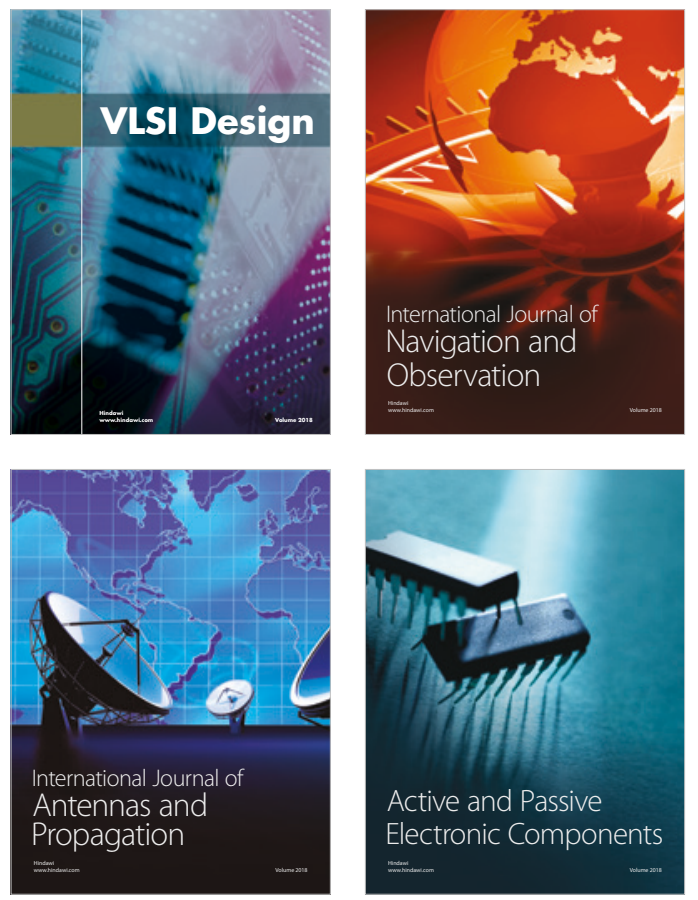
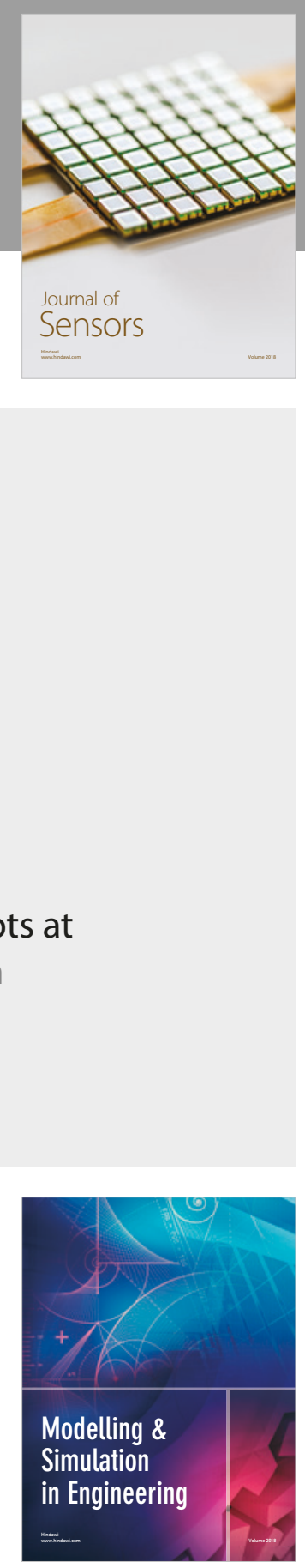

\section{Advances \\ Multimedia}
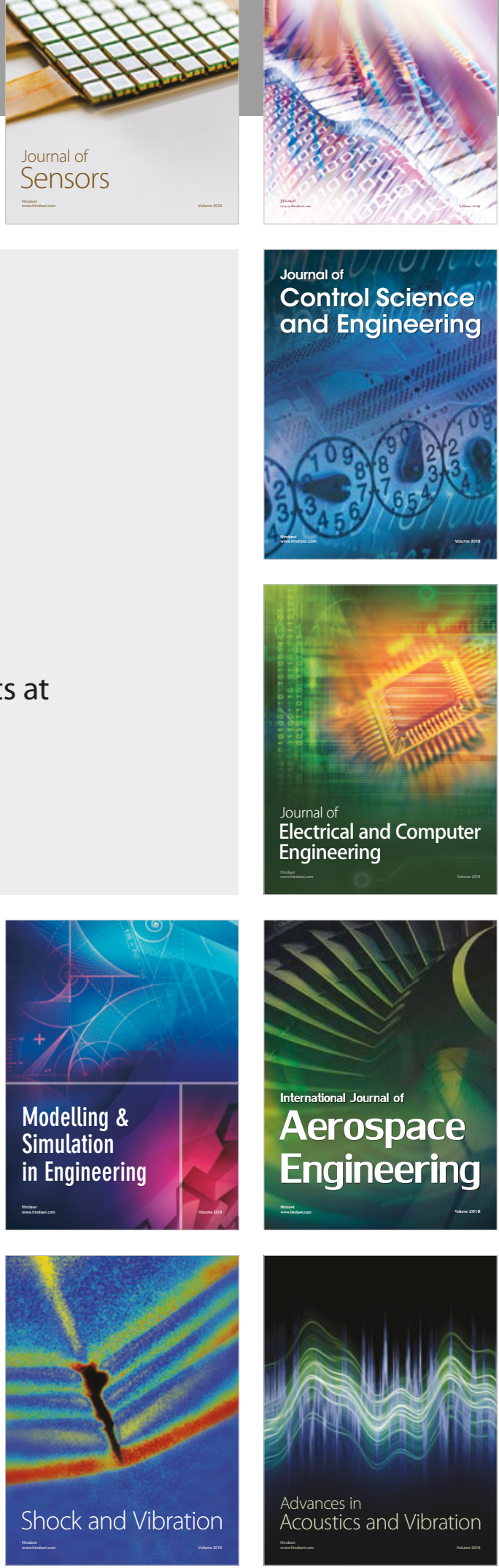